\title{
西藏聂拉木县色龙西山二裂-三纪 牙形石动物群及二叠一三叠系的分界
}

\author{
姚建新李子舜 \\ (中国地质科学院地质研究所, 北京)
}

世界二叠-三叠系之交的动物群都处在演化的低潮期和更替阶段,牙形石作为一种海相动 物群也不例外,但是同其他一些生物类群相比, 它有比较清楚的演化系列。这对于说明二叠一 三叠系之交生物类群的演化, 确立一条合乎生物地层学原理的二叠-三叠系界线至关重要. 因 此笔者对处在冈瓦纳古陆南缘的我国西藏南部地区的牙形石动物群及二叠-三叠系分界问题 进行了研究,得到一些认识报道如下:

\section{一、聂拉木县色龙西山牙形石动物群(图 1)}

西藏聂拉木县色龙西山是我国 Otoceras 层和中、下三叠统发育较好的地区之一. 在这里, Otoceras 层假整合于含四射珊瑚和海百合茎的二叠系色龙群之上, 仅厚 $0.2 \mathrm{~m}$, 为灰, 黄灰色白 云质灰岩, 富产菊不 Otoceras latilobatum Wang 等. 其上是 $6 \mathrm{~m}$ 厚的下三叠统灰色灰岩, 内含 菊石 Ophiceras (Lytophiceras) sakuntala Dieneri, Gyronites cf. superior Waagen), Anasibirites kingianu; (Waagen), Procarnites cf. kokeni (Arthaber) 等. 中三叠统为黄灰色,灰色灰岩夹灰 绿色砂岩和页岩,厚 30 余米,产菊石 Japonites meridianus Welter 等 ${ }^{[1]}$.

1982 年笔者和刘桂芳同志在色龙西山系统地采集了从 Otoceras 层到中三叠统的牙形石 样品,分析后获大量牙形石化石, 经鉴定共计有 8 属 19 种(图 2), 自下而上可划分出 8 个牙形 石带 (表 1)，其分别可和国内外的牙形石带进行对比，含义如下:

\section{Gondolella subcarinata changxingensis-Gondolella deflect 组合带 本带} 以 Gondolella subcarinata changxingensis Wang \& Z. H. Wang, G. deflecta Wang \& Z. H. Wang 为特征分子, 以 Anchignathodus parvus Kozur \& Pjatakova 出现为顶界, 常见的分子还 有 Gondolella planata (Clark), G. carinata (Clark), Ellisonia teicherti Sweet, Anchignathodus minutus (Ellison) 等. 该带大体相当于 Otoceras latilobatum 菊石带下部. Gondolella subcarinata changxingensis, G. deflecta 两个种最早见于我国浙江煤山长兴阶中. 这次我们直接从聂拉木 县色龙西山 Otoceras 层下半部分分析出大量 G. subcarinata changxingensis, G. deflecta, 而且 还未见典型三叠纪牙形石化石. 因此笔者将这两个种作为 Otoceras 层下半部分牙形石带的 代表分子. 此带还见于浙江长兴,四川广元等地。

2. Anchignathodus parvus 带本带以 Anchignathodus pardus Kozur \& Pjatakova 出现为底, 并以该种为本带特征分子, 此外还有 Gondolella carinata (Clark), G. planata

本文 1985 年 9 月 26 日收到。

第 1 期

科 学 通 报 


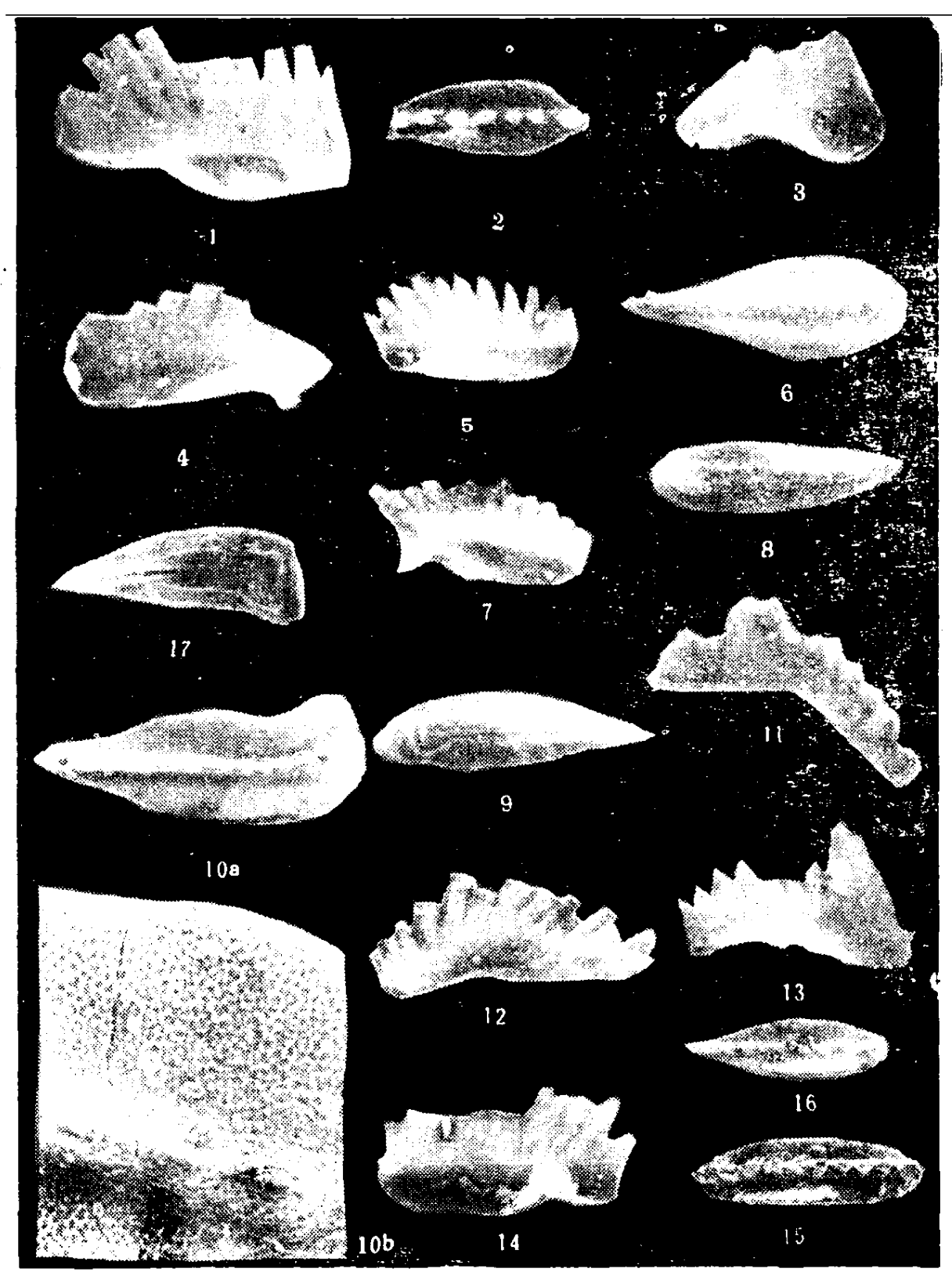

图 $\mathbf{1}$

1-1.鸡冠新齿牙形石 Neospathodus cristagalli (Huckriede) 侧视, $\times 100$; 西藏聂拉木县色龙西山， 下三丞统 Neosputhodus cristagalli 带. 采集号: Csl-30a, 登记号: 84261。1-2. 戈骨舟牙形石 Gondolella curinata (Clark) 口视， X90; 产地层位同上. 采集舅: Csl-30b, 登记号: 84506. 1-3. 微小近费然牙形石 Anchignathodus minutus (Ellison) 侧视, $\times 90$; 西藏聂拉木县色龙西山, Otoceras 层的 Gondolella sabcarinata changxingensis-G. deflecta 带. 采集号: Csli-6a, 登记号： 84277. 1-4. 香水新齿牙形石 Noospathodus xiangshuiensis Wang \& Daj 裸, X60; 下三统 Noospathodus. xiangshuiensis 带. 产地同上. 采集号: Csl-27c, 登记昂: 84254, 1-5, 14.再格新齿牙 形石 Neospazhodus waageni Sweet 5, 14，侧视，X48; 下三叠统 Neospathodus waageni 带.

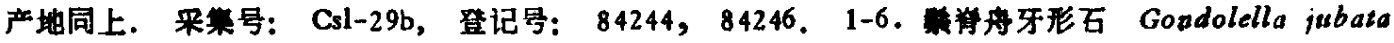
Sweet 口视，X60; 下三叠统 Gondolella jubata 带. 产地同上. 采集号: Csl-28b, 登记号: 84266 . 1-7. 迪内尔新铲形石 Neospathodus dieneri Sweet 侧视， $\times 60$; 下三统 Neospathodus dieneri 带. 产地同上. 采集号: Csl-312, 登记号: 84259.11-8, 16.亚龙骨舟牙形石长兴亚种 Gondolella subcarinata changxingensis Wang \& Z. H. Wang 8, 反口视, X60; 16, 口视，X90; Otoceras 层的 Gondolella subcarinata changxingensis-G. deflecta 带. 产地同上. 采集号: Cslj-6b, 登记 : 84504，84505. 1-9，15.冠辉舟牙形石 Gondolella regale Mosher 9, 反口视，X60；15，口枧， X90; 中三统 Gondolella regale 带. 产地同上. 采集号: Csl-24c, 登记号: 84507,84508. 1-10， 17. 偏斜舟牙形石 Gondolella deflecta Wang \& Z. H. Wang 10a, 10b, 口视, X48, X210; 17, 反 口视，X48; Otoceras 层的 Gondolella subcarinata changxingensis-G. deflecta 带. 产地同上. 采 集号: Cslj-6a, 登记号: 84270, 84271，84272. 1-11. 长梳频牙形石 Xaniognathus elongatus $\mathrm{Sw}$ :et

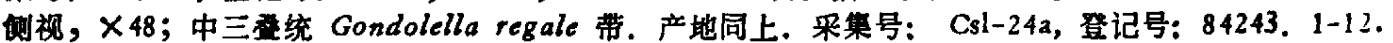

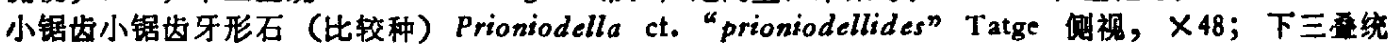
Neospathodus waageni 带. 产地同上. 采集号: Csl-29b，登记号：84250. 1-13.小近频齿牙形石 Anchignathodus parvus Kozur \& Pajatakova 调视, $\times 90$; Otoceras 层上部 Anchignathodus parvus 带. 产地同上. 采集号: Cslj-5b, 登记号: 84276. 所有标本都保存在中国地质科学院地质研究所 


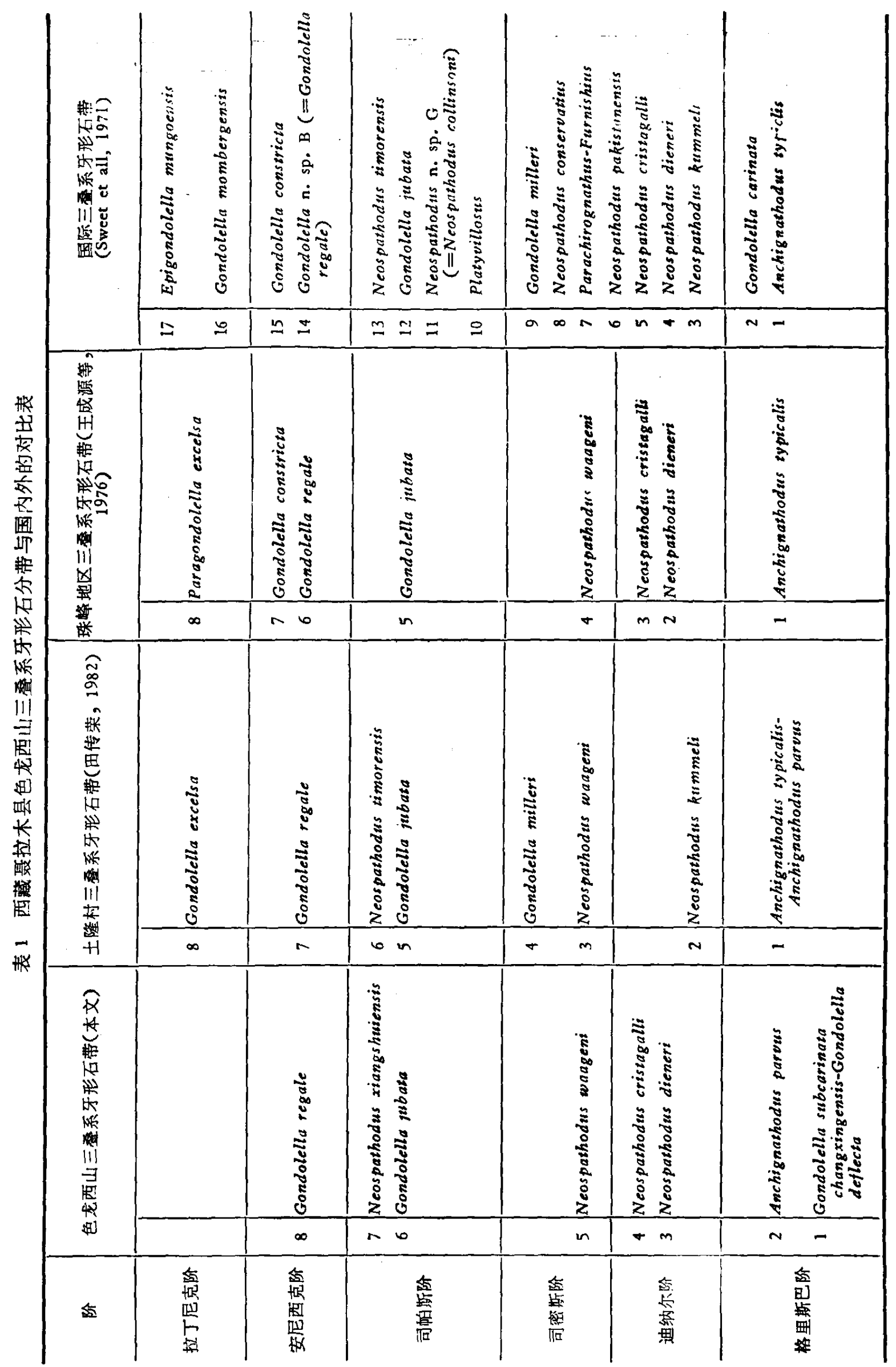




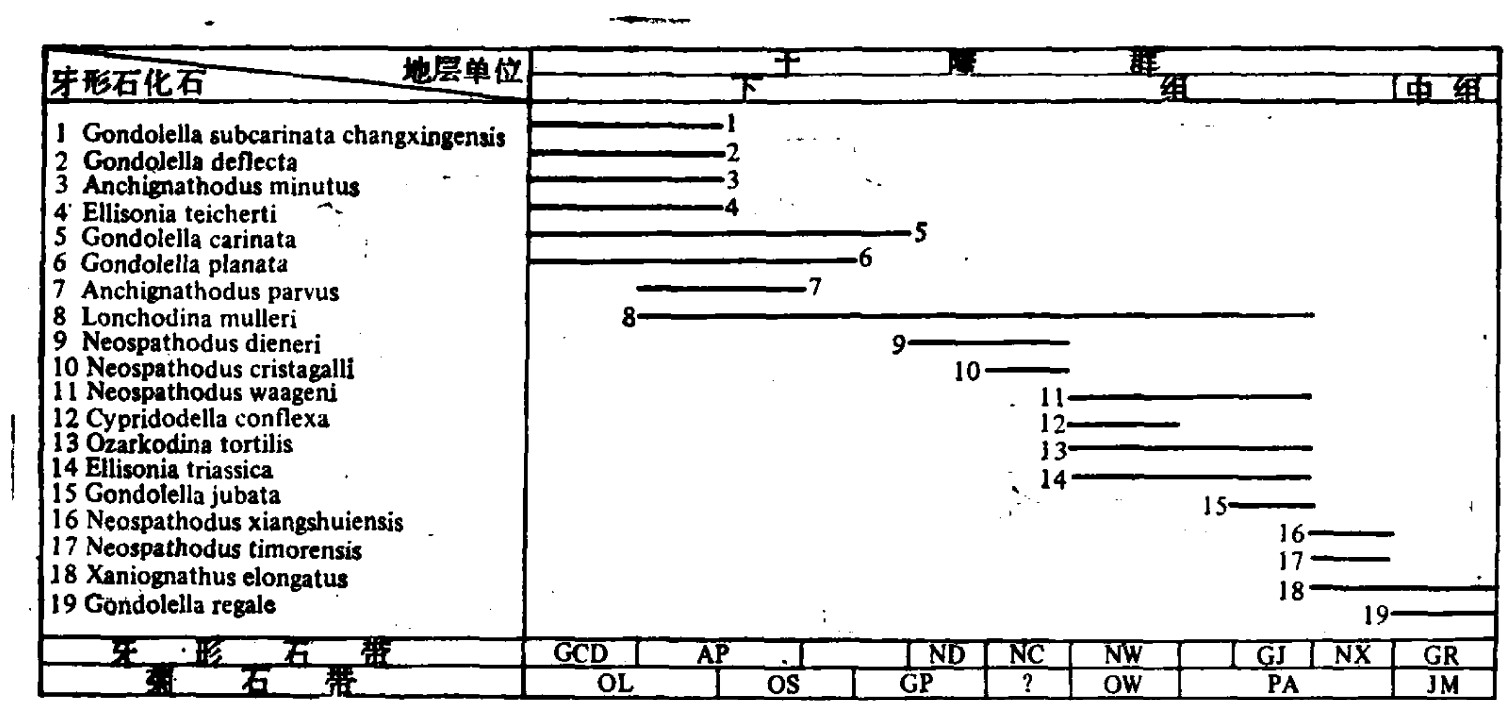

图 2 西藏聂拉木县色龙西山土隆群牙形石分布图

GCD Gondolella subcarinata changxingensis-G. deflecta zone, AP Anchignathodus parvus zone, ND Neospathodus dieneri zone, NC Neospathodus cristagalli zone, NW Neospathodus waageni zone, GJ Gondolella jubata zone, NX Neospathodus xiangshuiensis zone, GR Gondolella regale zone, OL Otoceras latilobatum zone, OS Ophiceras (Lytophiceras sakuntala zone, GP Gyronites psilogyrus zone, OW Owenites zone, PA Procarnites-Anasibirites zone, JM Japonites maguns zone

(Clark), G. subcarinata changixngnsis Wang \& Z. H. Wang, G. deflecta Wang \& Z. H. Wang, Lonchodina mulleri Tatge. 该带相当菊石 Otoceras latilobatum 带上半部分. A. parvus 是 我国飞仙关组最下部的牙形石化石. 在聂拉木县色龙西山, 它开始少量出现在 Otoceras 层上 半部分, 到 Ophiceras 层下部数量有明显增加. 该种在克什米尔,苏联和我国华南许多地区均 有发现.

3. Neospathodus dieneri 带本带以 Neospathodus dieneri Sweet 为特征分子, 另 外还有 Gondolella carinata (Clark). 该带位于下三叠统下部，相当 Sweet 牙形石第 4 带*, 与菊石 Gyronites cf. superior (Waagen) 相当, 时代为 Dienerian 早中期. 该带化石还见于巴 基斯坦西部、克什米尔.

4. Neospathodus oristagalli 带本带以 Neospathodus cristagalli (Huckried) 为 特征分子. 常见牙形石还有从下面延续上来的 $N$. dieneri Sweet. 该带相当 Sweet 牙形石 第 5 带,与菊石 Gyronites cf. superior (Waagen) 相当,时代为 Dienerian 中晚期. 该带化石 还见于我国西藏土隆、巴基斯坦西部、克什米尔等地.

5. Neospathodus waageni 带 本带以 Neospathodus wageni Sweet 为代表, 此外 还有 Ozarkodina tortilis Tatge, Gypridodella conflexa Mosher, Ellisonia triassica Muller 和 Prioniodella cf. “prioniodellides” Tatge.该带位于下三叠统中下部,相当 Sweet 牙形石第 7 带, 时代为 Smithian 早期. 该带化石还出现在我国西藏土隆、阿富汗、巴基斯坦、帝汶岛和北美.

6. Gondolella jubata 带 本带以 Gondolella jubata Sweet 为特征分子, 常见牙形石 有下部延续上来的 Neospathodus waageni Sweet, Ellisonia triassica Muller, Ozarkodina tortilis Tatge 等. 该带位于下三叠统上部, 相当于 Sweet 牙形石第 12 带, 㐘石 Procarinites-Anasibirites

* Sweet, W. C. 等,在总结三叠纪牙形石生物地层时,在巴基斯坦所建立的牙形石带 (1970). 
层上部,对代为 Spathian 中晚期. 该带化石还见于我国西藏土隆、巴基斯坦、希腊和北美等地。

\section{Neospathodus xiangshuiensis 带 本带以 Neospathodus xiangshuiensis Z. H.} Wang \& Dai 为代表分子, 另外还有 $N$. iimorensis (Nogami), Lonchodina mulleri Tatge 等。 该带位于下三叠统上部,大体相当于菊石 Procarinites-Anasibirites 层顶部, 时代为 Spathian 晚 期. N. xiangshuiensis Z. H. Wang \& Dai 最早见于我国四川江油，川北地区下三叠统嘉陵 江组第三段。在聂拉木县色龙西山下三叠统上部的地层中，N. xiangshuiensis Z. H. Wang \& Dai 很丰富,而 N. timorensis (Nogami) 的数量却很少，因此我们将 N. xiangshuiensis Z. H. Wang \& Dai 作为本带的代表分子.

8. Condolella regale 带 本带以 Gondolella regale Mosher 为特征分子，含有 Xaniognathus elongatus Sweet. G. regale Mosher 这个种是 Sweet 牙形石第 14 带的代表分 子. 该带位于中三叠统下部，与菊石 Japonites 相当,时代为 Anisian 早期. 此带见于我国西 藏土隆、美国北部等地。

\section{二、二骨-三桑系界线的探讨}

多年以来,许多地质工作者围绕着二叠一三叠系界线的划分问题做了大量工作. 目前关于 界线的划分大体有两种意见. 1. 主张采用传统界线，将两系界线置于 Otoceras woodwardi 带 底部，把过渡层中的二叠纪型分子作为子遗处理 ${ }^{[2]}$. 2. 主张将 Otoceras 层归人上二叠统, 认 为 Otoceras 层的生物组合基本上是二叠纪面貌 ${ }^{[3]}$.

传统界线实质上是一条生物绝灭的界线,古生代的䉇类,四射珊瑚和三叶虫以及棱菊石类 等都消失在这条界线之下. 但是一些二叠纪型的非簉有孔虫、海相介形类和腕足动物中的长 身贝类和戟贝类却延伸到了这条界线之上. Otoceras 层上界实质上是一条一些类群新生和一 些类群大量繁盛的界线. 如一部分介形虫 (Londaia) 和牙形石 (Isarcicella) 新生及克 氏蛤、蛇宷石类大量繁盛. 从这条界线起“三 叠型”分子占了主导地位. 如果考虑以大量 新生为主要分界的话,我们认为,第二种划分 意见更为合理一些,其理由如下。

中、古生代之交生物界发生了很大变化， 生物的更替量达 $50 \%-75 \%$ 。但是一些资 料表明,在二叠、三叠两纪之交，生物界的交 替并非同时发生的(图 3)。古生代动物群分 子的消失连续发生在上二叠统内. 从早二叠 世晚期到早三叠世早期这样短的时间内，古 老动物群分子的绝灭比率远大于新分子出现 的比率 ${ }^{[4]}$. 如: 在我国华南地区四射珊瑚的 复杂类型—一复体三带型——在中石炭世到 早二叠世达到极盛,晚二叠世早期衰退,至晚

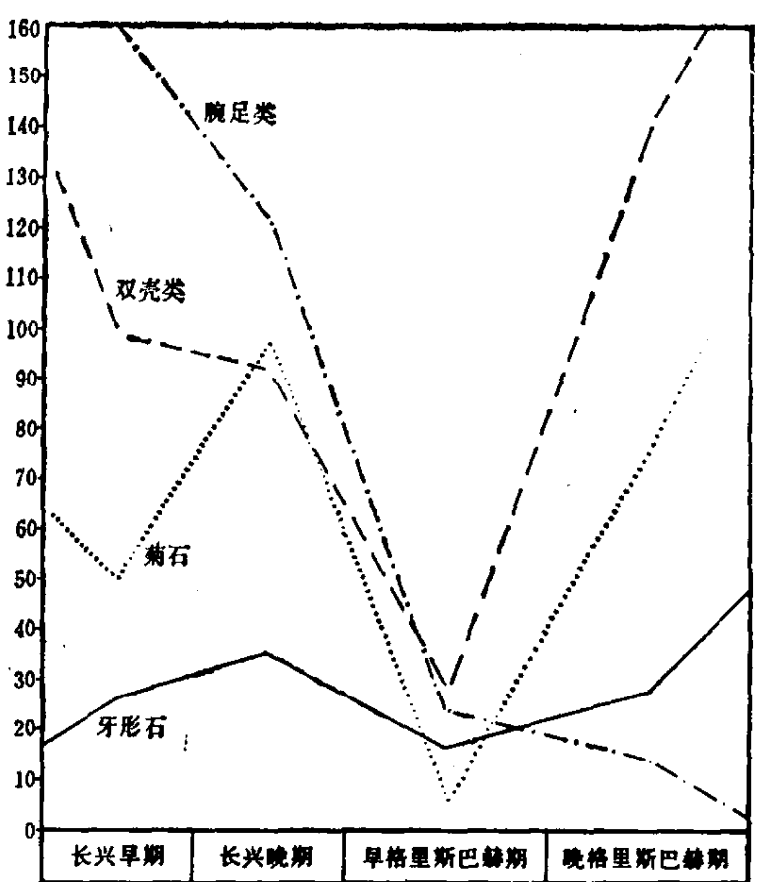

图 3 华南二叠-三叠系界线附近主要生物门类变化图 二叠世晚期 (长兴期) 只剩下个别属，而且以单体的单带或双带的简单类型的小型珊瑚为主. 晚古生代腕足类的石燕类、长身贝类和扭月贝类在晚二叠世早期先后出现一些特化种类，如 
Leptedus, Oldhamina, Perigeyerella 等,然后相继绝灭, 剩下一些小型原始类型延人三叠纪中. 腹足类从晚二量世开始就被原始的类型神螺类占了主要地位，在长兴期出现了一些特化类型， 如 Extandilabrum, Aiptospira，但它们在二叠纪内相继绝灭，越过界线的只是神螺类中体型小， 壳管较简单的那些种。双壳类亦相似,据海扇超科的统计,在早、晚二叠世之交发生大的衰退， 属数锐减三分之一匹].

从上例事实可以看出, 那些对环境反映敏感的类型常随着环境改变而衰退和绝灭．在二 叠一叠系之交半咸水的浅水相动物群变化最明显. 陆相(包括淡水相)生物变化也同样明显. 狭盐性底栖生物受绝灭的影响较大. 浮游生物, 广盐性浅水底栖生物受绝灭影响并不大. 在 中、下三叠统没有或很少有狭盐浅水底栖生物出现,而在深水环境中，其动物群一般没有什么 变化 ${ }^{[4]}$. 综上所述,我们看到，仅以某些类型的生物绝灭来确定两系的界线是不合适的. 因为 这种绝灭并不能真正反映出生物群的演化规律，而且很难找到一条几乎所有门类都同时绝灭 的界线. 我们认为以新的生物类群的大量出现和䇣盛作为一个新时代开始的标志才比较合 理.

我国西藏聂拉木县色龙西山牙形石动物群的分布可以证明，新的牙形石分子 Anchigna. thodus parvus 出现是在 Otoceras 层的上部, 而进人 Ophiceras 层后，Anchignathodus parvus 才开始大量的繁盛. Oteceras 层下半部分的 Gondolella subcarinata changxingensis-Gondolella deflecta 带中，我国浙江煤山长兴阶上部牙形石带的代表分子 ${ }^{[6]}$ G. subcarinata changxingensis 和 G. deflecta 非常丰富. 本带中的其他分子基本上也是古生代的类型. 这样,在色龙西山晚 二叠世的牙形石延续到了传统上认为属于三叠系底部的 Otoceras 层中. 相似的情况也发现 于斯匹梯的 Otoceras 层 ${ }^{[7]}$ 和我国四川广元的 Hypophiceras 层 ${ }^{[8]}$ 中. Bhatt 等研究了斯匹梯的 Otoceras 层牙形石后认为: “Otoceras 层是以平台型牙形石 Gendolella orientalis Barskov \& Korolcva 和 G. subcarinata (Sweet) 为特征，根据最近牙形石生物地层的演化标准看，它们 是二叠世地层中的类型 (Sweet, 1973；Kozur, $1974 \& 1977$ ), Otoceras 层不应是三叠系的 底而应是二叠系的顶。” 巴特的意见有一定的合理性. 从色龙西山牙形石动物群的特征看， Otoceras 层下部的 Gondolella subcarinata changxingensis-G. deflecta 带的时代也应属于二叠 世.

另外, 东特提斯和世界其他地区二叠一三叠纪地层和牙形石的新近研究也表明 Otoceras woodwardi 带的上部的牙形石动物群很明显要比伊朗西北和苏联外高加索 Paratirolizes 动物 群时代要新. 但是 Otoceras woodwardi 中的牙形石动物群还具有二叠纪的特征. Gondolellla orientalis Barskov \& Koroleva 和 G. subcarinata (Sweet) 在 Otoceras woodwardi 中依然存在, 直到 Otoceras woodwardi 带顶部它们才消失. 在 O. woodwardi 带中没有见到典型的下三叠 统的牙形石属 Neospathodus Mosher 的分子. 只有在 ophiceras 动物群中它们才开始出现 ${ }^{\text {[? }}$. Kozur 研究的德国盆地三叠系底部地层最新资料显示出德国班砂统底部的动物群确实要比 Otoceras woodwardi 带时代要新, 甚至可能要比 ophiceras commune 带还要新, 因此在德国盆 地与 Otoceras 动物群相当的地层决不会包含在三叠系中 ${ }^{[?]}$.

此外，我国华南的研究资料也表明，大量新的生物类群的相对繁盛和辐射性发展不是在 Otoceras 层而是从 Ophiceras 层才开始的（图 3)。双壳类中的克氏蛤在二叠系中只有零星的 分布,在过渡层中也不多，从王氏假克氏蛤带开始，仅在西南三省就几乎同时出现了十七个种. 这些种大多数延续至下统更高层位。蛇菊石类在二叠纪只有一个有疑问的属(Douvilleoceras) 
至格里斯巴赫期爆发式地出现了近十个属及亚属, 并由此演变出三叠纪全部菊石类. 介形虫 中的郎岱介也是从王氏假克氏蛤带开始发生 ${ }^{[9]}$. 在 Otoceras 层或 Hypophiceras 层下部牙形 石仍以长兴阶的 Gondolella subcarinata, Gondolella deflect 为主. Anchignathedus parvus 从 Otoceras 层上部开始出现到 Ophiceras 层演化出它的特化类型 Isarcicella isarcica, 到 Dienerian 阶中, Neospathodus 属中的牙形石开始大发展,成为中、下三叠统中占统治地位的类型. 参考 上述情况, 我们认为将二叠一三叠系界线置于 Ophiceras 层底部的意见是较合理的. 这条界线 标志了在新时代开始时生物界的新生和辐射性发展.

\section{三、结 论}

1. 西藏色龙西山从 Otoceras 层到中三叠统一共可以划分出 8 个牙形石带。这些带分别 可和国内外相当的牙形石带进行对比.

2. 古生代生物群的绝灭连续发生在晚二叠世内, 很难找到一条共同的绝灭界线, 而且这种 绝灭是与一定的生物相有关系的.

3. 从牙形石和其他一些生物门类新类型的出现和辐射性演化看, 二叠一三叠系界线应在 Ophiceras 层底部, 也就是 Claraia wangi 带底部或 Isarcicella isarcica 带底部.

11] 尹集祥等,珠穆朗玛峰地区科学考察报告, 1966-1968, 地质,科学出版社, 1974, $81-126$.

: 2] Sheng, et al., Jout. Fac. Sci., Hokkaido Univ., Ser. IV, 21 (1984), 1:133-181.

[3] Gupta, V. J. and Kozur, H., Stratigraphy and Structure of Kashmir and Ladakh Himalaya Contributions to Himalayan Geology, 2 (1983), $292-298$.

i 4 ] Kozur, H., Freibeiger Forshungsheft, C 326(1977), 73-86.

[5] 尉鸿福等,国际交流地质学术论文集 1,地质出版社, 1984, 195-202.

[6]王成源、王志浩,中国微体古生物学会第一次学术会议论文选集,科学出版社, 1979, 114-120.

[7] Bhatt, D. K. et al., Joul. Palacont. Soc. India, 25(1981), 130-134.

[8] 张景华等,国际交流地质学术论文集 1, 地质出版社, 1984, 163-178.

[9] Bando, Y. et al., Recent Reserches in Geology, 8(1982), 1-54. 